\title{
An Intelligent Continuous Collection Device for High-Pressure Flow Synthesis: Design and Implementation
}

\section{Uninterrupted Smart Diversion in High Pressure Synthesis}

Supporting Information

Michael Tilley, Guanlong Li, Paul Savel, Debasis Mallik, Michael G. Organ*

Department of Chemistry, York University, 4700 Keele Street, Toronto, Ontario, Canada, M3J 1P3

* organ@yorku.ca 


\section{Table of Contents}

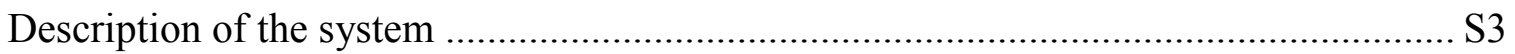

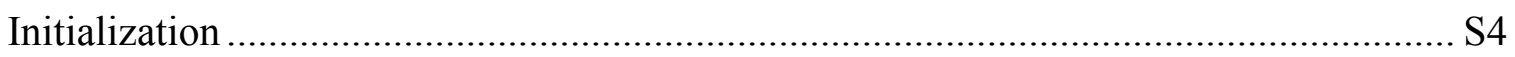

Validation of fluid dynamics in collection loops........................................................ S6

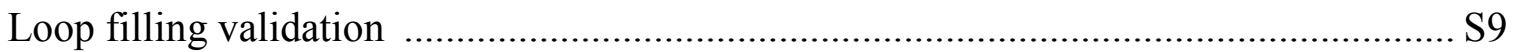

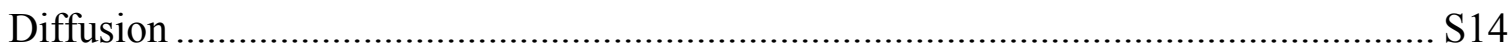

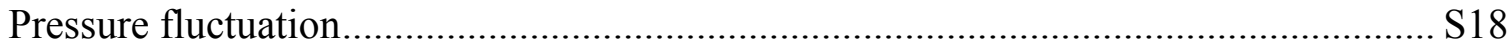

Pressure fluctuation at collection valve .............................................................. S19

Operation of Continuous Collection Device......................................................... S20

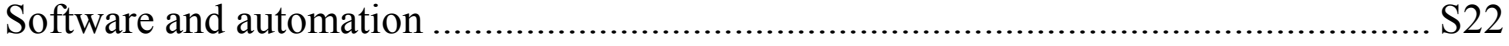

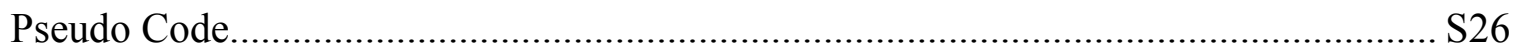

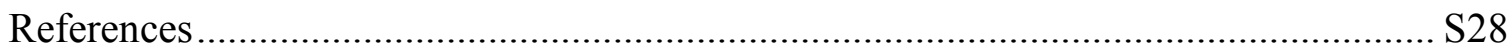




\section{Figures and Tables}

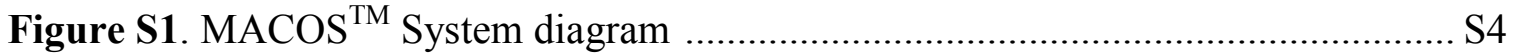

Figure S2. Example of Maestro software set up .................................................. S6

Figure S3. Dispersion model for laminar flow in circular tubes ................................ S7

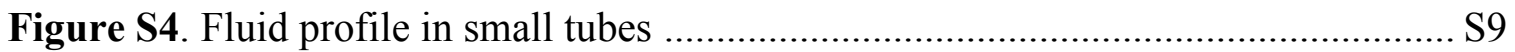

Figure S5. Reproducibility of dispersion effect in diversion loop at $600 \mu \mathrm{L} / \mathrm{min}$......... S1 1

Figure S6. Clean-out of diversion loop, $1500 \mu \mathrm{L}$ fill, 3000ppm, $100 \mu \mathrm{L} / \mathrm{min} \quad \ldots \ldots \ldots \ldots . . . . \mathrm{S} 12$

Figure S7. Clean-out of diversion loop, $1500 \mu \mathrm{L}$ fill, $3000 \mathrm{ppm}, 5000 \mu \mathrm{L} / \mathrm{min} \quad \ldots \ldots \ldots . . . \mathrm{S} 13$

Figure S8. Clean-out of diversion loop, $1500 \mu \mathrm{L}$ fill, $100000 \mathrm{ppm}, 5000 \mu \mathrm{L} / \mathrm{min} \quad \ldots \ldots . . \mathrm{S} 14$

Figure S9. Normalized diffusion plot for $\mathrm{D}=10^{-7} \mathrm{~m}^{2} / \mathrm{s}$ in small tube ....................... S16

Figure S10. Diffusion of 3000 ppm APE in MeOH in 0.04" ID tube ....................... S17

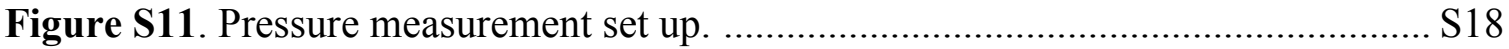

Figure S12. Linear relationship between pressure and voltage ................................. S19

Figure S13. Pressure fluctuation during diversion valve actuation .......................... S20

Figure S14. Coordination of sampling valve, V2, and collection valve, V3 .............. S21

Figure S15. Schematic of MACOS ${ }^{\mathrm{TM}}$ workflow for smart diversion highlighting communication between synthesis and analysis units $\mathrm{S} 25$

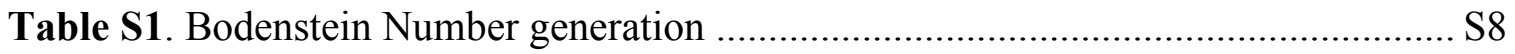




\section{Description of the System}

The MACOS ${ }^{\mathrm{TM}}$ system consists of two main units: the synthesis unit, where high temperature, high pressure reactions are performed, and the analytical unit, where the results of these reactions are characterized. The main process line of the synthesis unit consists of two Harvard Apparatus High Force 70-2202 syringe pumps equipped with 2.5mL stainless steel syringes (model\# 70-2269), high pressure Vici Valco Cheminert valves (C2 series), and one piston pump, Vici Valco M50, which pumps transfer line fluid under atmospheric pressure along with fittings from Swagelok and Vici Valco Inc. The two Harvard pumps are combined with a 4-port valve (C2-2004EH) to make a continuous flow unit (CFU) delivers a continuous flow of reaction material into the process line. The syringes of the CFU are loaded with fluorinated buffer to prevent reagent from contacting with syringe. ${ }^{1}$ The flow passes through a 6-port valve (C22006EH) for sampling, and an 8-port valve for collection (C2-2008EH). The 6 port valve is equipped with a $5 \mu \mathrm{L}$ sample loop. The 8-port valve has two $2000 \mu \mathrm{L}$ loops for collecting process material. The piston pump delivers solvent, and pushes the content of the collection valve to either the product vessel or the waste vessel. The structure of the full system is shown in Figure S1. All parts are controlled by a custom circuit board and software that were designed in house.

The analytical unit includes a Gerstel MultiPurpose Sampler (MPS workstation) dual arm liquid handler and an Agilent 1260 Infinity HPLC. Coordination between the two devices is handled by Maestro software which supports sample preparation, as well as creation and execution of analytical sequences. 


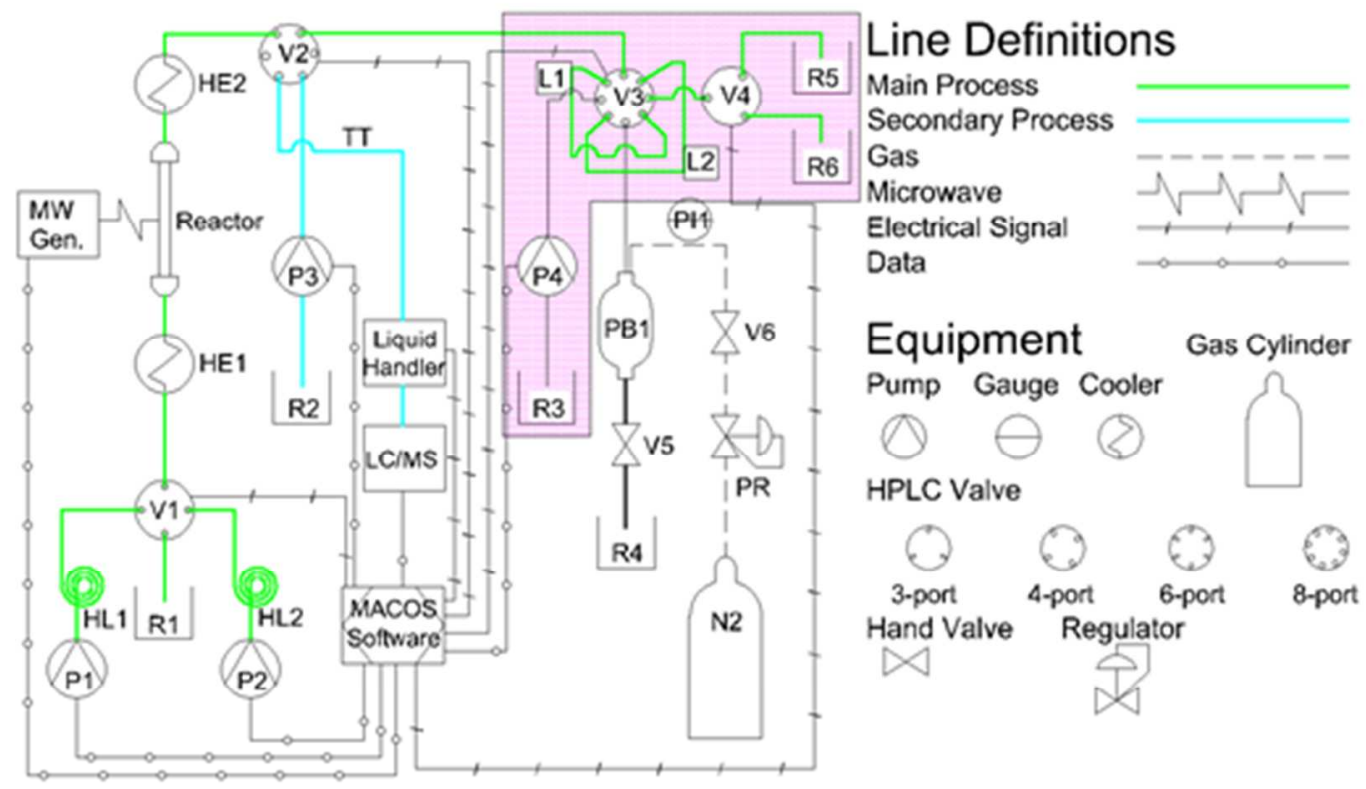

Figure S1. MACOS $^{\mathrm{TM}}$ System diagram. P1 \& P2: syringe pump, P3 \& P4: transfer solvent pump, L1 \& L2: reagent holding loop, R1: reagent reservoir, R2: sample transfer solvent reservoir, R3: collection transfer solvent reservoir, R4: Transfer solvent collection reservoir, R5: product collection reservoir, R6: waste collection reservoir, V1: continuous flow unit valve, V2: sampling valve, V3: continuous collection valve, V4: diversion valve, V5: manual pressure release valve, V6: manual gas shut off valve, HE: heat exchanger, PB: pressure ballast, PI: Pressure indicator, PR: pressure regulator, TT: sample transfer tube.

\section{Initialization}

Prior to the experiment, the CFU is loaded and primed and the software parameters are input. 
To load or reload the CFU, the operator is prompted through a series of steps. Upon executing the routine, the software will empty and refill each syringe in turn. The operator is prompted to place a waste vessel at location R1 and hit the enter key when this is done, the first syringe then pumps out any residual material. Next, the operator is prompted to place the new reagent vessel at $\mathrm{R} 1$. Upon acknowledgement, the syringe refills with this material, then the 4-port CFU valve toggles so that the second syringe is in communication with $\mathrm{R} 1$. The CFU is loaded after repeating the same steps on the second syringe. Note nothing goes into the process line during this step.

Priming is an automated routine. To prime the process line, the system outlet valve (V5, Figure $\mathrm{S} 1$ ) is open, so that the system remains at atmospheric pressure. The CFU is set to run twice the system volume. During the run, the valves in the process line are toggled according to their loop sizes in order to make sure the air is evacuated from all tubing. Once the priming routine is complete, the system is pressurized by the operator; V5 is closed and the system is pressurized by opening the pressurized gas line valve, V6.

Software must be set up for both the synthesis and analysis units. The software for the synthesis unit is readied by entering the parameters of the process, including flow rate, sampling rate, reaction temperature and total volume of process material. The analytical unit is setup from Maestro by loading a prep-sequence of liquid handling instructions for a process sample. Figure S2-a) shows an example of the prep-sequence. The "wait" and "output" lines are used to coordinate with the synthetic unit of MACOS ${ }^{\mathrm{TM}}$. This will be described further in the "software and automation" section. Maestro is also used to manage the sequence of the samples (Figure S2-b) to be analyzed and the analytical method to be used. 
(a)

\begin{tabular}{|c|c|c|c|c|c|c|}
\hline Action & MPS & Method /Value & Source & Vial & Destination & Vial \\
\hline (2) WAIT & Right MPS & test & & & & \\
\hline DILUTE & Left MPS & mt_Guan & & & Waste3 & \\
\hline$\zeta$ OUTPUT & Right MPS & mt3 & & & & \\
\hline - WAIT & Right MPS & test & & & & \\
\hline OSILUTE & Left MPS & $\mathrm{m} \cdot 2$ & & & Tray1,VT98f & \\
\hline$\$$ OUTPUT & Right MPS & $m+3$ & & & & \\
\hline OWAIT & Right MPS & test & & & & \\
\hline TOILUTE & Left MPS & mt_Guan2 & & & Waste3 & \\
\hline \ OUTPUT & Right MPS & $\mathrm{mt3}$ & & & & \\
\hline
\end{tabular}

(b)

\begin{tabular}{|c|c|c|c|c|c|c|c|c|}
\hline & Sample Name & Method & Tray & Vial & Volume & Sample Info & Flename & Type \\
\hline 1 & 10Port & 00_JK_10PORT.M & Tray1, vT98f & 97 & 5.0 & BLK & BLK_ACN & Sample \\
\hline 2 & 10Port & 00_JK_10PORT.M & Tray1, VT98f & 71 & 5.0 & Sample & E4A_PAA_1T1_1 & Sample \\
\hline 3 & 10Port & 00_JK_10PORT.M & Tray1,VT98f & 72 & 5.0 & Sample & E4A_PAA_1T1_2 & Sample \\
\hline 4 & 10Port & 00_JK_1OPORT.M & Tray1,VT98f & 73 & 5.0 & sample & E4A_PAA_1T1_3 & Sample \\
\hline 5 & 10Port & 00_JK_10PORT.M & Tray1,vT98f & 74 & 5.0 & sample & E4A_PAA_1T1_4 & Sample \\
\hline 6 & 10Port & 00_JK_1OPORT.M & Tray1,vT98f & 75 & 5.0 & sample & E4A_PAA_1T1_5 & Sample \\
\hline 7 & 10Port & 00_JK_1OPORT.M & Tray1,VT98f & 76 & 5.0 & sample & E4A_PAA_1T1_6 & Sample \\
\hline 8 & 10Port & 00_JK_10PORT.M & Tray 1 , VT98f & 77 & 5.0 & sample & E4A_PAA_1T1_7 & Sample \\
\hline 9 & 10Port & 00_JK_10PORT.M & Tray1, vT98f & 78 & 5.0 & sample & E4A_PAA_1T1_8 & Sample \\
\hline 10 & 10Port & 00_JK_1OPORT.M & Tray1, vT98f & 79 & 5.0 & sample & E4A_PAA_1T1_9 & Sample \\
\hline
\end{tabular}

Figure S2. Example of Maestro software set up. a) prep-sequence b) sample sequence

\section{Validation of Fluid Dynamics in Collection Loops}

The loops on the diversion valve uses 0.04" ID stainless steel tubing. Dispersion of the laminar flow profile was modeled analytically following the work of Taylor, ${ }^{2}$ Aris $^{3}$ and the Jensen group. ${ }^{4}$ In particular, we used a modified Bodenstein Number $(B o)$ :

$$
B o=\frac{4 \beta D \tau}{d_{t}^{2}}
$$

where $\beta$ is the shape factor (48 for circular tubes), $D$ is the diffusion coefficient, $\tau$ is the residence period in the tube in question (an amalgam of flow rate and flow area), $d_{t}$ is the tube inner diameter. The larger $B o$, the more the flow resembles an ideal laminar convection model, with characteristic parabolic dispersion profile: 


$$
v(r)=v_{m}\left[1-\frac{r^{2}}{R^{2}}\right]
$$

This standard equation relates the flow velocity at a given radial position $v(r)$ to the midline (maximum) velocity $v_{m}$ as a ratio of radial distances, $\frac{r^{2}}{R^{2}}$, generating the familiar parabolic shape of the laminar flow regime. Conversely, the smaller $B o$, the more pluglike the flow profile. Using a "typical" liquid phase diffusion coefficient of $10^{-9} \mathrm{~m}^{2} / \mathrm{s}$, Jensen et al. charted dispersion effects, see Figure S3, below.

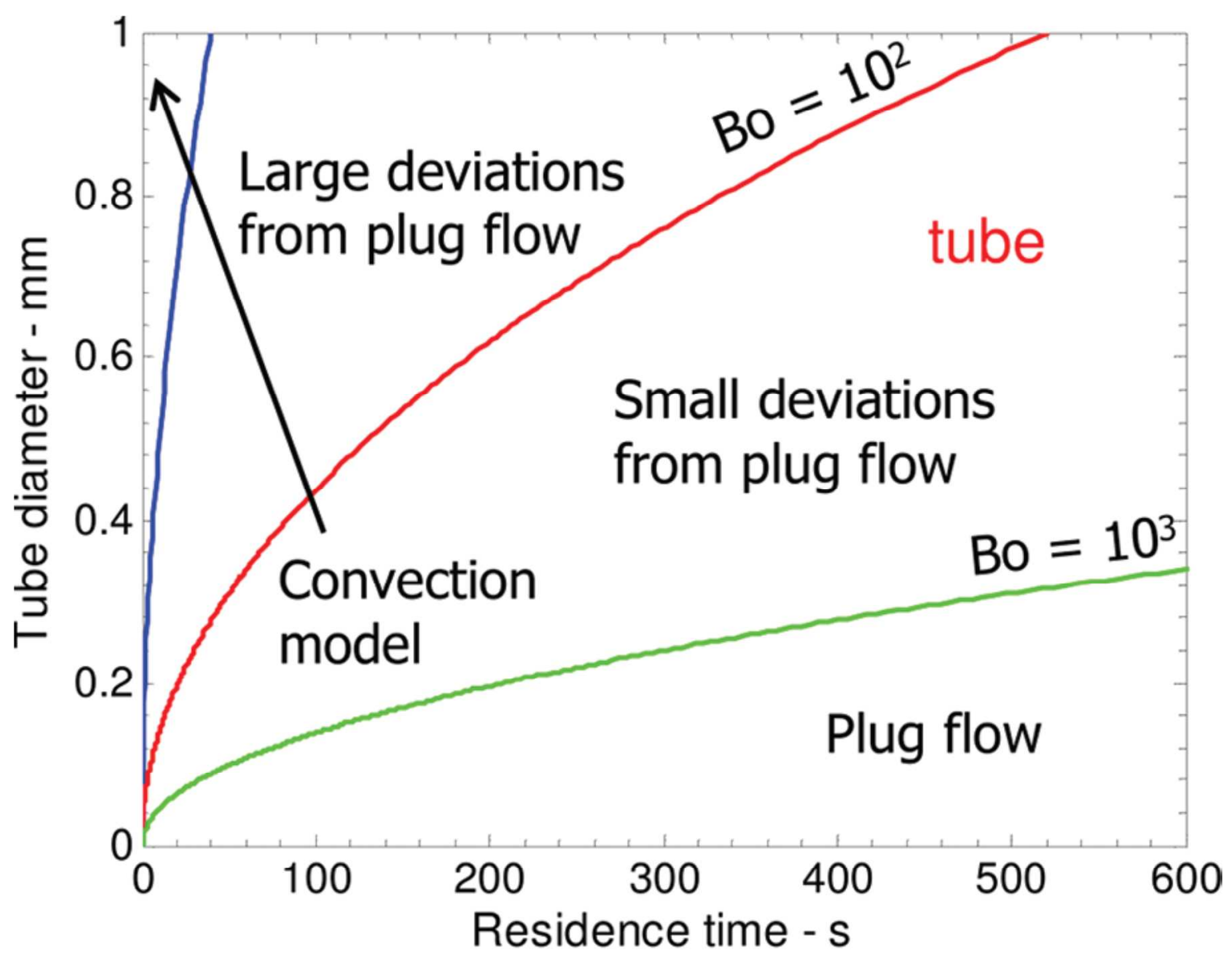

Figure S3. Dispersion model for laminar flow in circular tubes. ${ }^{4}$

Jensen et al. made three distinctions in dispersion effects as they relate to $B o$ for a given $D$ and $\tau$ to predict the flow profile. $B o>1000$ is indicative of plug flow regimes, wherein 
cohesive effects of the fluid largely overcome the dynamic shear forces resulting in a more or less flat interface. With velocity increase, diameter decrease, and diffusivity decrease, $B o$ drops indicating deviation from plug flow. For $100<B o<1000$, the deviation is relatively small. For $B o<100$ the deviation is large, and for $B o<10$, the flow may be characterized as an ideal laminar flow. We employed these useful heuristics for the analytical evaluation of the $\operatorname{MACOS}^{\mathrm{TM}}$ collection system (see Table S1).

Applying the modified Bodenstein number (S1) to our parameters (loop volume of 2000 $\mu \mathrm{L}$, inner diameter 0.04 " (1.02 $\mathrm{mm}$ ), flow rates $1 \mu \mathrm{L} / \mathrm{min}$ to $1000 \mu \mathrm{L} / \mathrm{min}$, and diffusivity from $10^{-11} \mathrm{~m}^{2} \mathrm{~s}$ to $10^{-7} \mathrm{~m}^{2} / \mathrm{s}$ ), the following results were obtained:

\begin{tabular}{|c|c|c|c|c|c|c|c|}
\hline $\begin{array}{c}\text { Flow Rate }(\mu \mathrm{L} / \mathrm{min}) \\
/ \text { Diffusivity }\left(\mathrm{m}^{2} / \mathrm{s}\right)\end{array}$ & 1 & 10 & 20 & 100 & 200 & 500 & 1000 \\
\hline $10^{-11}$ & 221 & 22 & 11 & 2 & 1 & 0 & 0 \\
\hline $10^{-10}$ & 2215 & 221 & 111 & 22 & 11 & 4 & 2 \\
\hline $10^{-9}$ & 22145 & 2215 & 1107 & 221 & 111 & 44 & 22 \\
\hline $10^{-8}$ & 221453 & 22145 & 11073 & 2215 & 1107 & 443 & 221 \\
\hline $10^{-7}$ & 2214533 & 221453 & 110727 & 22145 & 11073 & 4429 & 2215 \\
\hline
\end{tabular}

Table S1. Bodenstein Number generation. This table demonstrates that for a given set of system dimensions, Bo decreases (tendency to laminar flow) with higher flow rates and lower diffusivity. ${ }^{6}$ Green indicates plug flow conditions, red indicates "small" deviations from plug flow, blue, "large" deviations from plug flow, yellow, laminar convection, according to the Jensen group distinctions.

Broadly speaking, within the parameters of the $\operatorname{MACOS}^{\mathrm{TM}}$ flow system, plug flow is predicted for slower flow rates, with deviations expected to become significant around $200 \mu \mathrm{L} / \mathrm{min}$ leading to more ideal laminar type flow at the high end of the flow rate $(500 \mu \mathrm{L} / \mathrm{min}$ ). Loop filling validation tests were performed at $600 \mu \mathrm{L} / \mathrm{min}$ (to ensure 
compliance from maximum system flow rate limits, described above), while system performance tests central to the diversion operation were performed at 50 and $100 \mu \mathrm{L} / \mathrm{min}$ in order to reduce solvent waste.

\section{Loop Filling Validation}

Validation tests were performed on the Continuous Diversion Device (CDD) 8-port loops to determine the maximum nominal fill capacity. As dispersion effects increase at higher laminar flow rates, it is important to understand if the parabolic flow profile produced would cause fluid to extend beyond the nominal fluid front as defined by the metered volume, as in Figure S4. Dispersion of the magnitude of this example would reduce the loss of reaction products as actuating the CDD valve would cut off the tip of the fluid front.

(a)

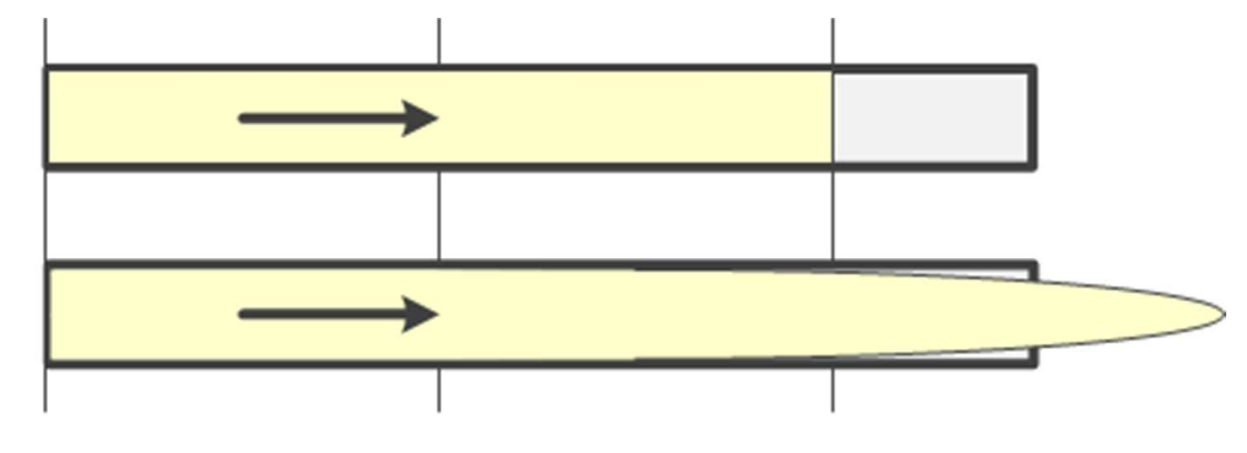

$0 \mathrm{uL}$

$50 \mathrm{uL}$

$100 \mathrm{uL}$

Figure S4. Flow profiles in small tubes. a) Plug flow profile b) Laminar, parabolic flow profile.

In order to determine the extent of dispersion in the diversion loop within $\mathrm{MACOS}^{\mathrm{TM}}$ operational parameters, $1500 \mu \mathrm{L}$ of analyte solution was flowed into the loop of the 
collection valve (V3) at $600 \mu \mathrm{L} / \mathrm{min}$. The valve was switched and loop was then flushed in the same direction with transfer solvent, at $100 \mu \mathrm{L} / \mathrm{min}$. Successive $100 \mu \mathrm{L}$ fractions were captured to determine the extent of dispersion. $0 \mu \mathrm{L}$ represents the loop inlet, the $2000 \mu \mathrm{L}$ mark represents the loop outlet. Figure S5 shows results of loop filling capacity tests. Fractions from $1600 \mu \mathrm{L}$ to $2000 \mu \mathrm{L}$ are shown to demonstrate the extent of dispersion beyond the nominal flowed test volume. At $600 \mu \mathrm{L} / \mathrm{min}$ the fractions show a pronounced parabolic curve, extending approximately $500 \mu \mathrm{L}$, or $33 \%$ beyond the bulk flow. This parabolic protrusion is less pronounced than the "ideal" profile, which would extend twice the length of the bulk volume in a fully developed regime, suggesting either that laminar flow has not fully developed over the length of the loop or that surface tension forces within the fluid act to counter the shear forces acting upon it. Nevertheless, it is a significant deviation from plug flow and must be accounted for when determining collection parameters, as product will be lost by having the parabolic nose protrude from the far end of the loop. The main conclusion to be drawn by is that within operational limits the dispersion is limited to $1 / 3$ the nominal volume at the highest flow rate. We have defined $1500 \mu \mathrm{L}$ as a maximum volume for reproducibly filling the collection loop at flow rates up to $500 \mu \mathrm{L} / \mathrm{min}$. 


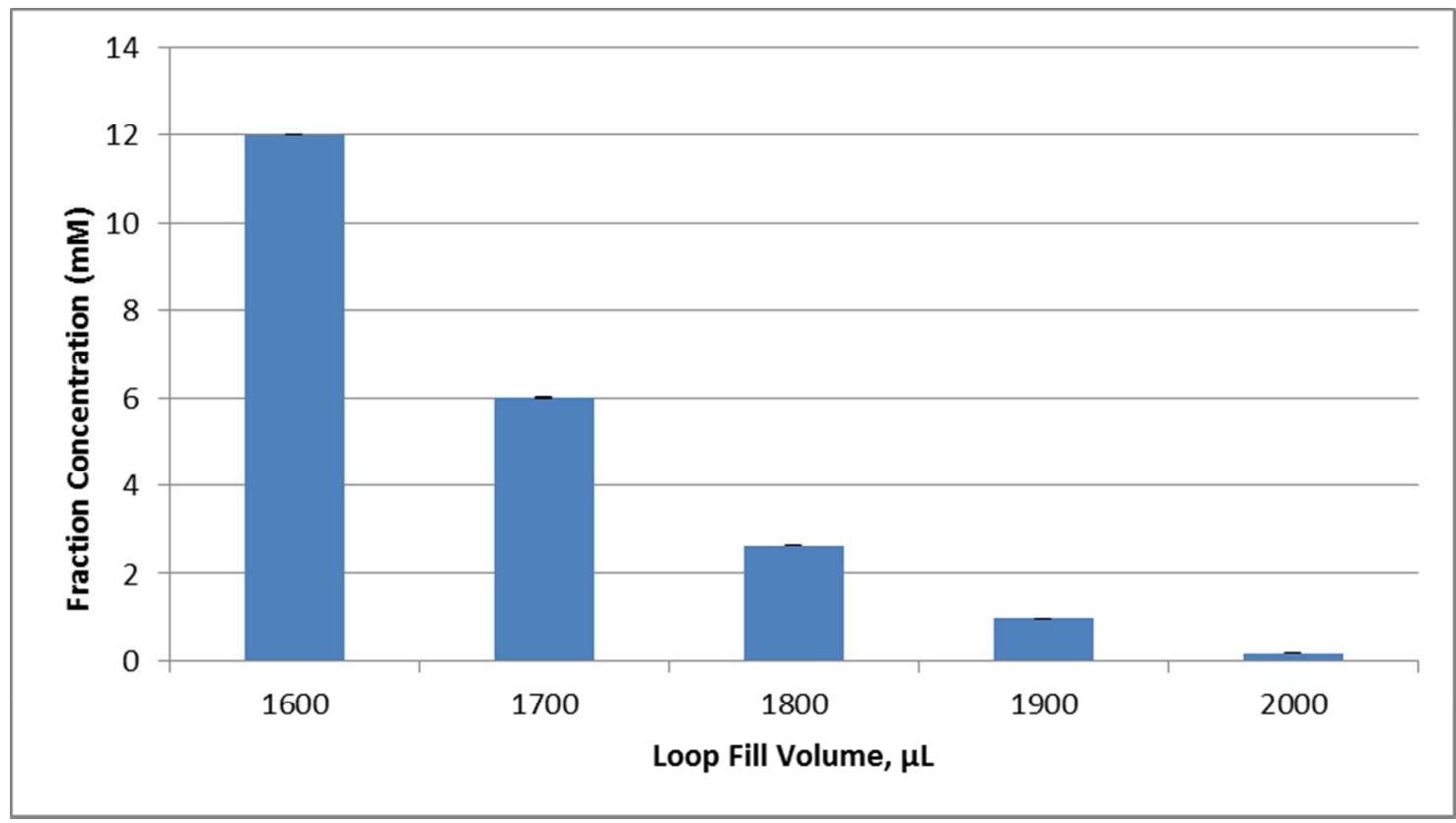

Figure S5. Reproducibility of dispersion effect in diversion loop for $600 \mu \mathrm{L} / \mathrm{min}$ flow rate.

Accurate filling of the collection loop ensures no loss of the product of interest. The ability to produce discrete micro-lot volumes requires the complete elimination of carryover. We proceeded to examine how thoroughly the diversion loop could be cleaned in preparation for the next fill cycle. Using the operational values, $1500 \mu \mathrm{L}$ of $3000 \mathrm{ppm}$ (22.4mM) allyl phenyl ether in $\mathrm{MeOH}$ was pumped into the loops at $100 \mu \mathrm{L} / \mathrm{min}$. The loop was then evacuated using pure $\mathrm{MeOH}$ at $100 \mu \mathrm{L} / \mathrm{min}$, with fractions collected every $500 \mu \mathrm{L}$. The resulting graph, Figure S6, shows a characteristic curve reproduced over 3 runs, wherein the analyte is flushed out as a slightly distended plug. No analyte is found in the first fraction, the next 5 or 6 fractions contain the total analyte volume. Beyond the $6^{\text {th }}$ fraction, no analyte was observed during any run. Average recovered analyte volume was $94 \%$; it is unlikely to expect $100 \%$ recovery due to sample processing uncertainties, including a multiple dilution required to bring the samples into calibration range, and the 
sensitivity of the calibration curve. Therefore the total volume of solvent required to clean the loop and collect $1500 \mu \mathrm{L}$ of $3000 \mathrm{ppm}$ allyl phenyl ether in $\mathrm{MeOH}$ is $3 \mathrm{~mL}$.

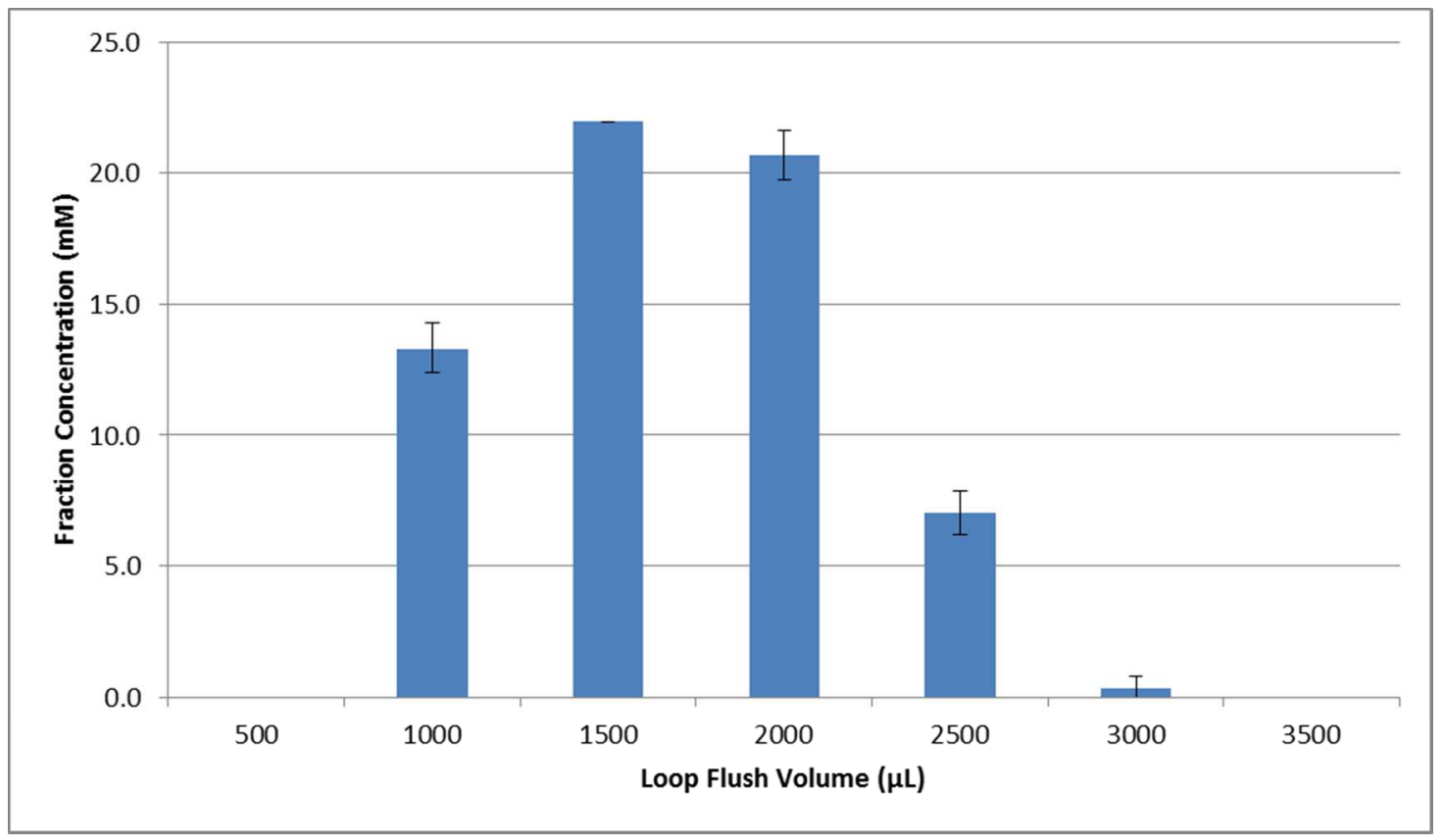

Figure S6. Clean-out of diversion loop, $1500 \mu \mathrm{L}$ fill, 3000ppm, $100 \mu \mathrm{L} / \mathrm{min}$.

Cleanout efficiency was also checked against higher cleaning solvent flow rates, as typical operation conditions require flow rates greater than $100 \mu \mathrm{L} / \mathrm{min}$. Figure $\mathrm{S} 7$ below shows a trial using a $5000 \mu \mathrm{L} / \mathrm{min}$ flow rate. The cleanout curve is virtually identical, and recovered analyte volume was $92 \%$, indicating that speed of loop flush does not significantly affect efficiency. 


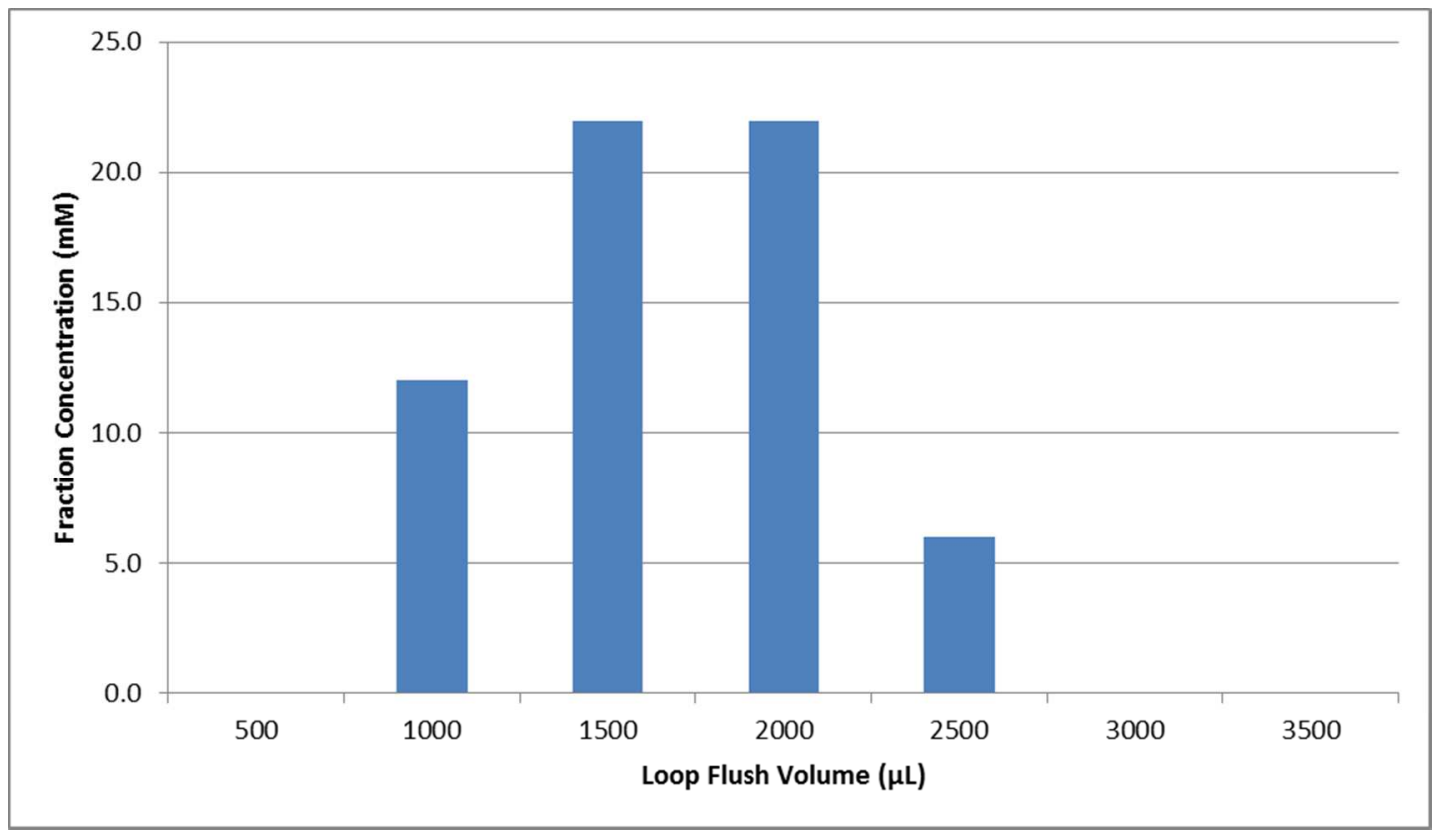

Figure S7. Clean-out of diversion loop, $1500 \mu \mathrm{L}$ fill, 3000ppm, $5000 \mu \mathrm{L} / \mathrm{min}$.

A trial using $100000 \mathrm{ppm}(0.75 \mathrm{M})$ and $5000 \mu \mathrm{L} / \mathrm{min}$ solvent pump flow rate was run (Figure S8) to validate clean-out of more concentrated solutions. Again, this shows a similar curve, and recovered analyte volume of $92 \%$ (below) within 6 fractions $(3 \mathrm{~mL})$ of transfer fluid. No significant differences are observed from the lower flow rate and lower concentration value experiments. This gives good confidence that the diversion procedure can be performed at normal processing rates without loss of micro-lot integrity. 


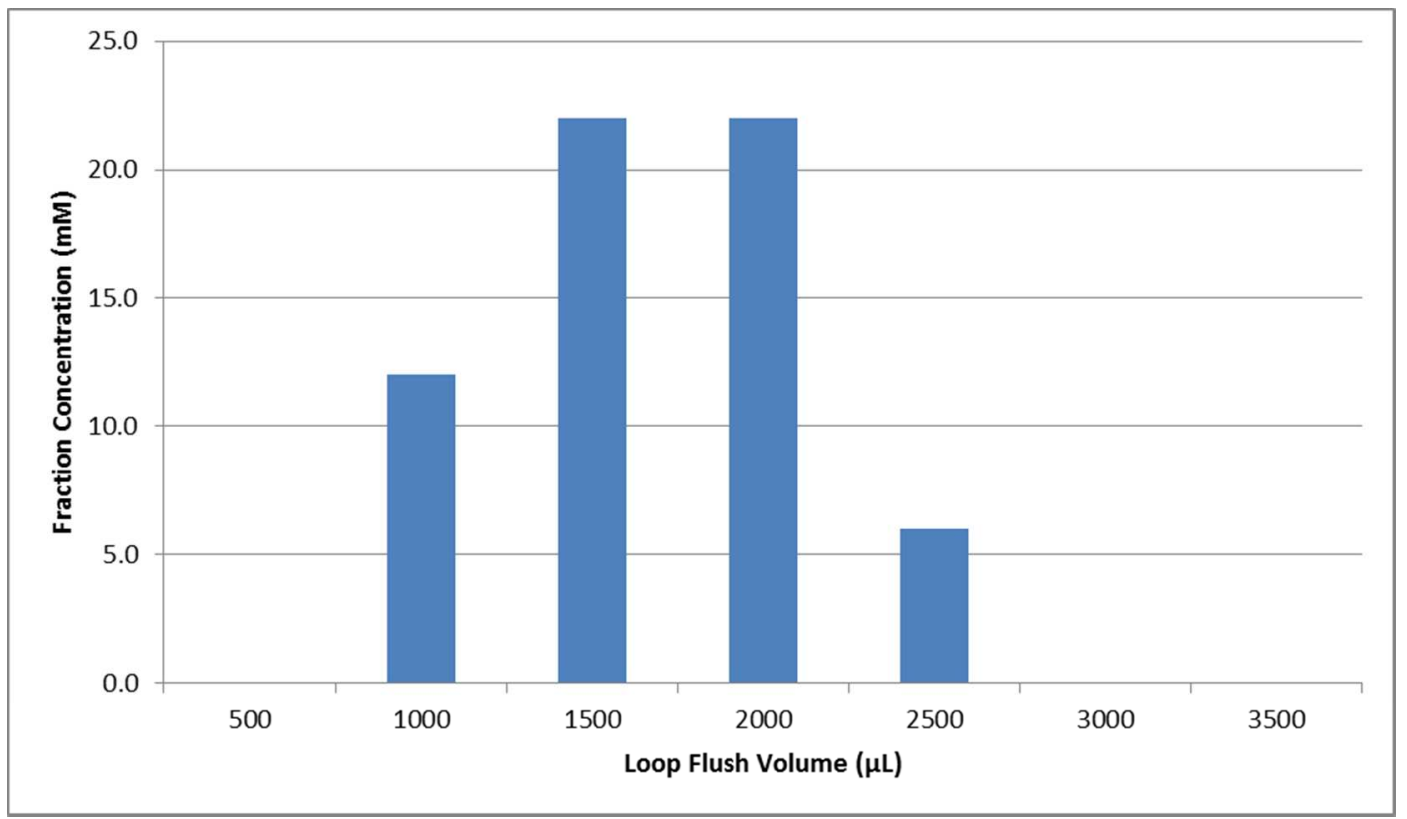

Figure S8. Clean-out of diversion loop, $1500 \mu \mathrm{L}$ fill, $100000 \mathrm{ppm}, 5000 \mu \mathrm{L} / \mathrm{min}$.

\section{Diffusion}

Consideration must be given to diffusion as well as dispersion, for at slower flow rates the sample resides in the diversion loop for a longer period of time. If diffusion adds to the extension of the analyte front, it is possible that some might escape the end of the loop before collection is finished. An analytical representation of the diffusion in a tube was produced by applying Fick's Second Law of Diffusion (Eq. S3) that predicts

$$
\frac{\delta \phi}{\delta t}=D \frac{\delta^{2} \phi}{\delta x^{2}}
$$

concentration $(\varphi)$ change of some solution with diffusivity (D) over time $(t)$ at a point $(x)$. In the present case (Figure S9) we considered the initial condition to be a flat interface of unitless concentration, 1 , over the cross section of tube, which will give a proportional 
map of the diffusion profile. As a result of the geometry, we can consider diffusion in this to be one-dimensional, with an infinite solute source (concentration at $x=0$ never decreases). Diffusivity is considered here at $10^{-7} \mathrm{~m}^{2} / \mathrm{s}$, two orders of magnitude greater than generally proposed ${ }^{4,5}$ to give a conservative basis for estimating the effects of even highly mobile solute particles, for instance if the solution was heated. From the plot, it would take approximately $3 \mathrm{~min}$ for the concentration $10 \mathrm{~mm}$ from the interface to rise to $10 \%$ of the interface value. It would take 2.5 hours for a point $80 \mathrm{~mm}$ away to achieve the same concentration rise. One would expect to see an $80 \mathrm{~mm}$ extension in the fluid front if collecting $1500 \mu \mathrm{L}$ at $10 \mu \mathrm{L} / \mathrm{min}$ (150 minutes residence time), however this would still stay within the $2000 \mu \mathrm{L}$ space of the loop, and can be addressed by collecting smaller micro-lot volumes at this flow rate. 


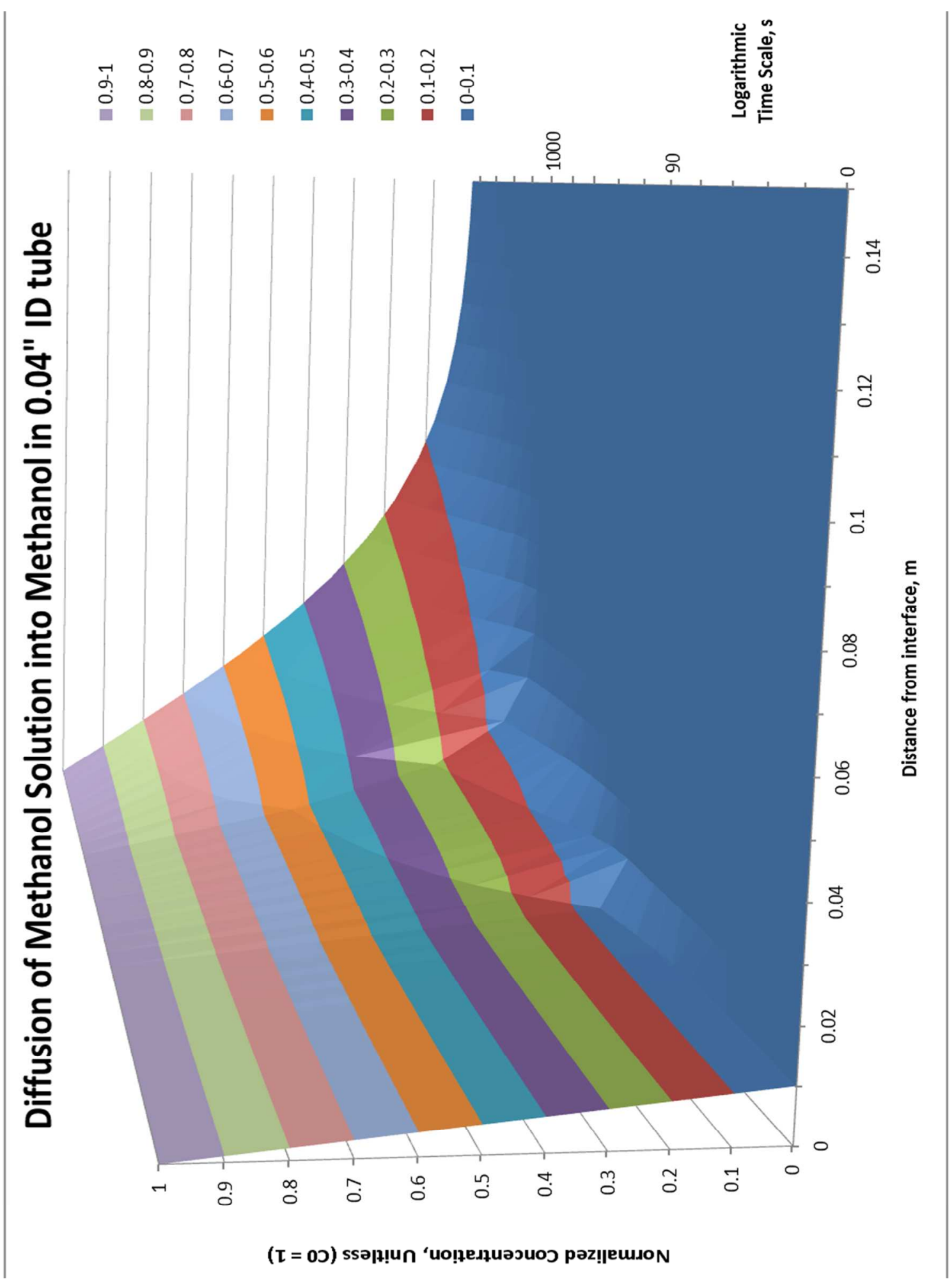

Figure S9. Normalized diffusion plot for $\mathrm{D}=10^{-7} \mathrm{~m}^{2} / \mathrm{s}$ in small tube. 
Experimental trials, illustrated in Figure S10 below, show $1700 \mu \mathrm{L}$ of $3000 \mathrm{ppm}$ analyte solution loaded at $100 \mu \mathrm{L} / \mathrm{min}$ and left for 1,3 , and $5 \mathrm{hrs} .100 \mu \mathrm{L}$ fractions were then pumped out in the same direction at $100 \mu \mathrm{L} / \mathrm{min}$ and analyzed.

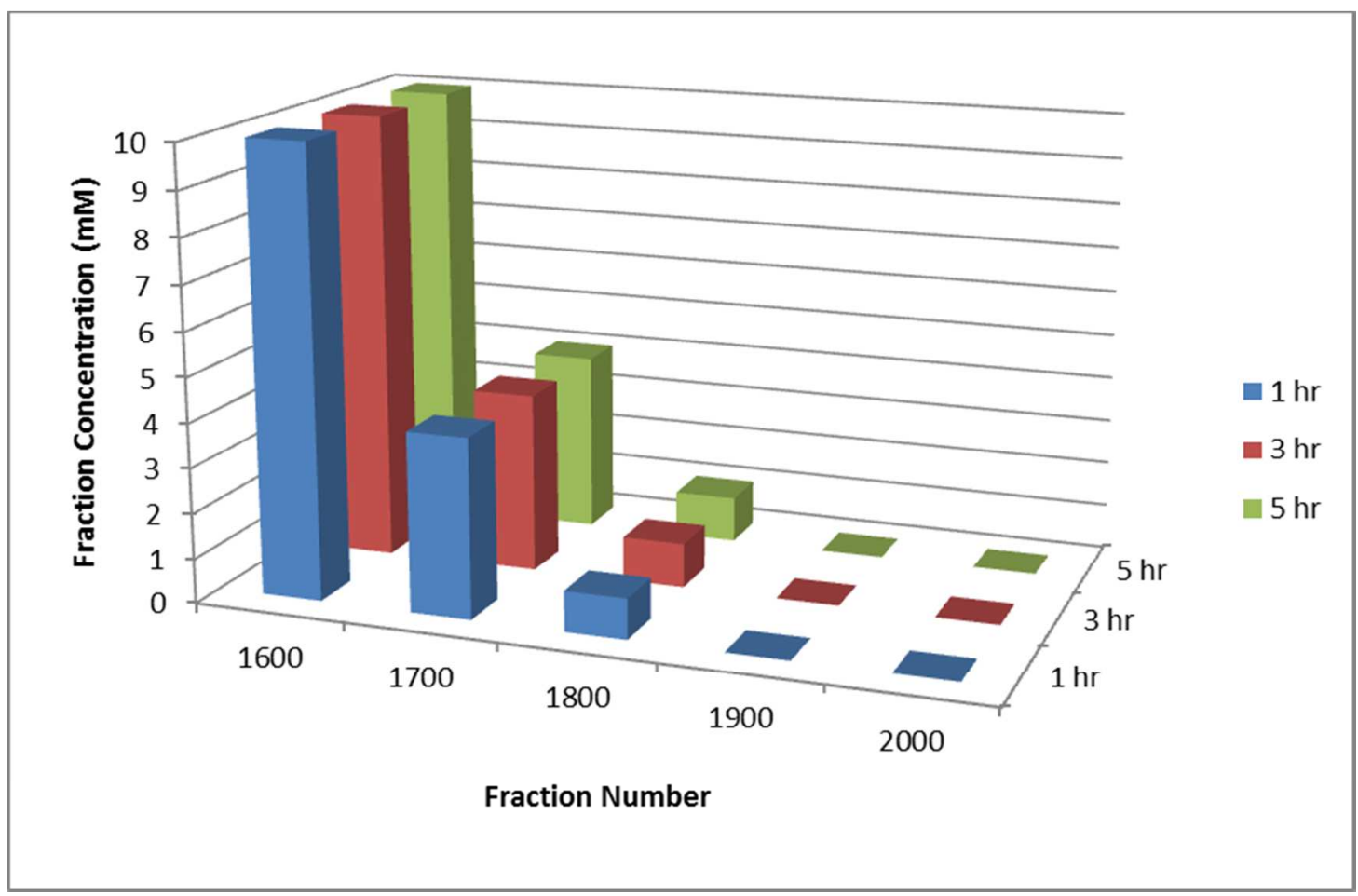

Figure S10. Diffusion of $3000 \mathrm{ppm}$ APE in $\mathrm{MeOH}$ in 0.04" ID tube.

No significant diffusion was observed in these runs. In fact, there is less diffusion than predicted in the calculations, quite possibly because the actual coefficient of diffusivity for the solution is less than the 'normal' $10^{-7} \mathrm{~m}^{2} / \mathrm{s}$ value most often cited, which would significantly reduce the predicted diffusion. It does not appear that in the timescale of the process, diffusion will have a significant effect. 


\section{Pressure fluctuation}

In order to ensure that the actuation of the collection valve does not disrupt the system pressure, the pressure fluctuation in the process line was measured using a transducer installed inline. The transducer takes pressure as its input, and converts it to voltage signals. Since the multimeter is not sensitive enough to detect small variations in the raw output voltages, a signal amplifier was added along with a resistor to boost the signal. This setup is illustrated in Figure S12 below, where the CFU schematic in lower-left corner shows the location of the pressure transducer during experiments.

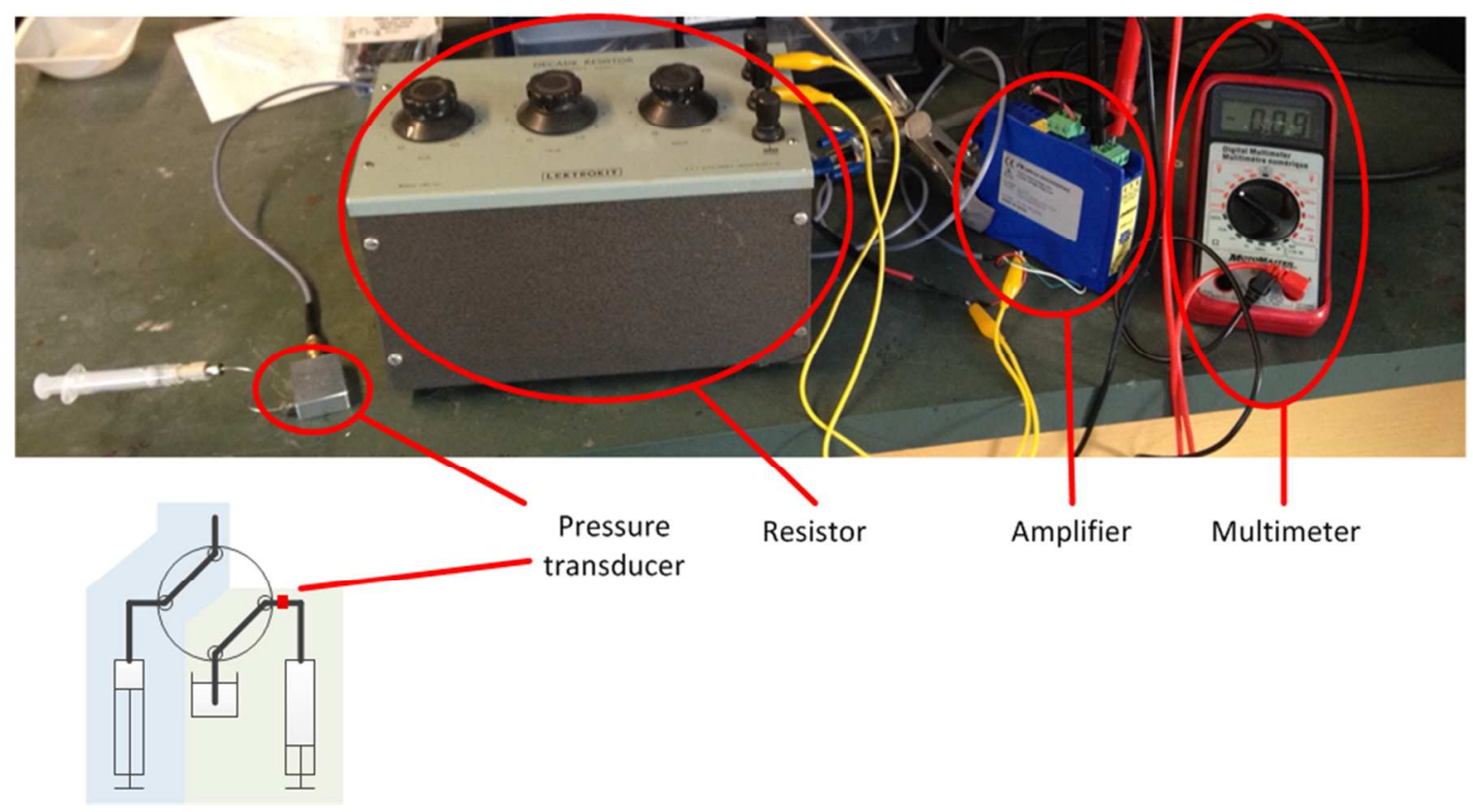

Figure S11. Pressure measurement set up.

Figure S13 shows the amplified transducer voltage calibrated against a gas pressure gauge reading. This plot shows a clear linear relationship between voltage and pressure. The results of the experiment show that the two variables are strongly related. 


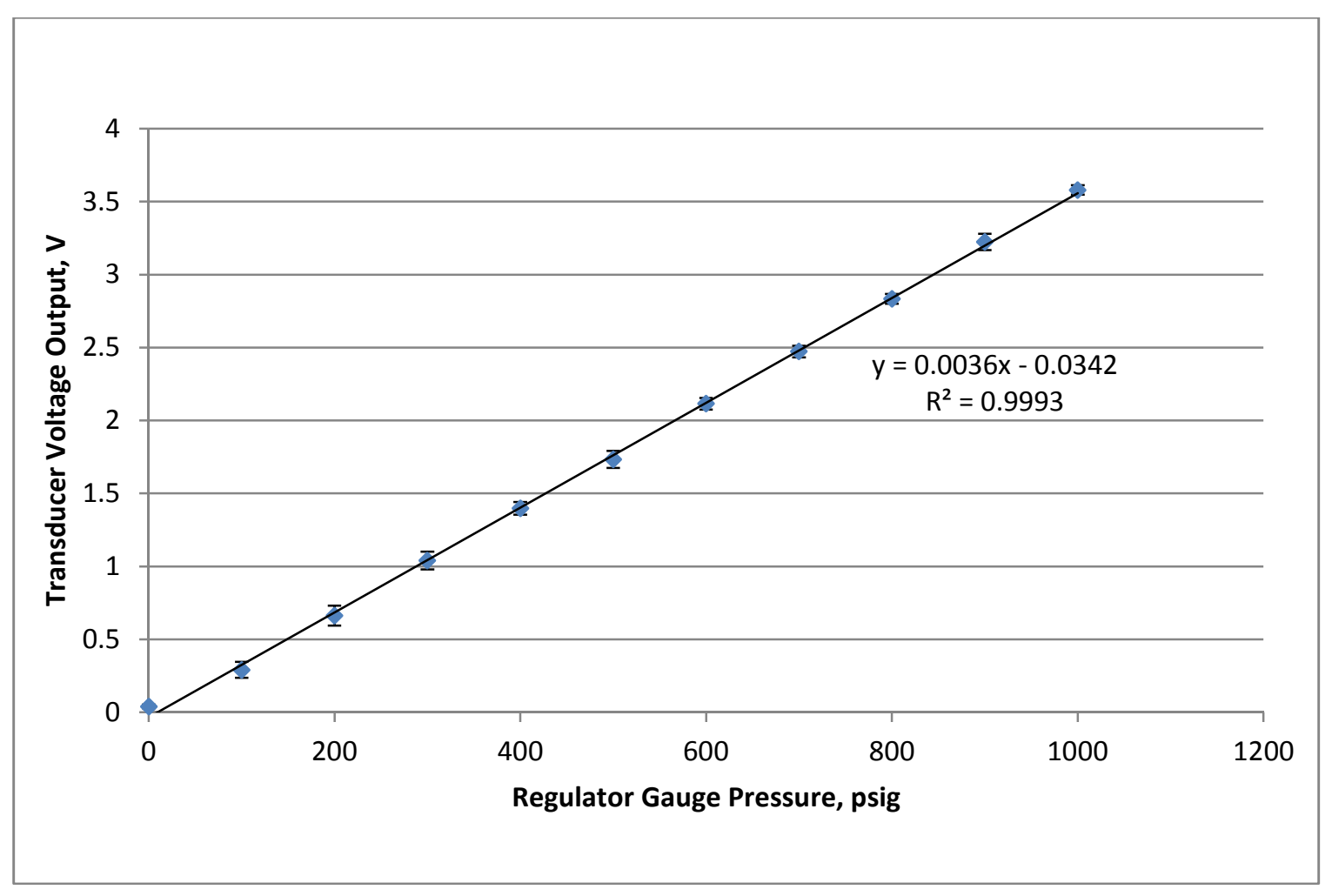

Figure S12. Linear relationship between pressure and voltage.

\section{Pressure Fluctuation at the Collection Valve}

Because the diversion valve communicates between two very different pressure regimes, pressure fluctuation was measured as the valve An experiment was set up to determine the pressure fluctuation in the system at $1000 \mathrm{psi}$. With the synthesis unit primed and pressurized and fluid flowing at a flow rate of $100 \mu \mathrm{L} / \mathrm{min}$ the diversion valve, $\mathrm{V} 3$, was set to switch every minute. The multimeter reading was observed during these actuations and voltage was converted back to pressure using the equation in Figure S13. As shown in Figure S14, the pressure fluctuation over 10 actuations averaged slightly less than 4 psi, $0.4 \%$ of 1000 psi system pressure, not a significant factor that would affect the process 
CPPs. Part of the fluctuation is likely the result of instrument overshoot because of the sudden, large changes in pressure.

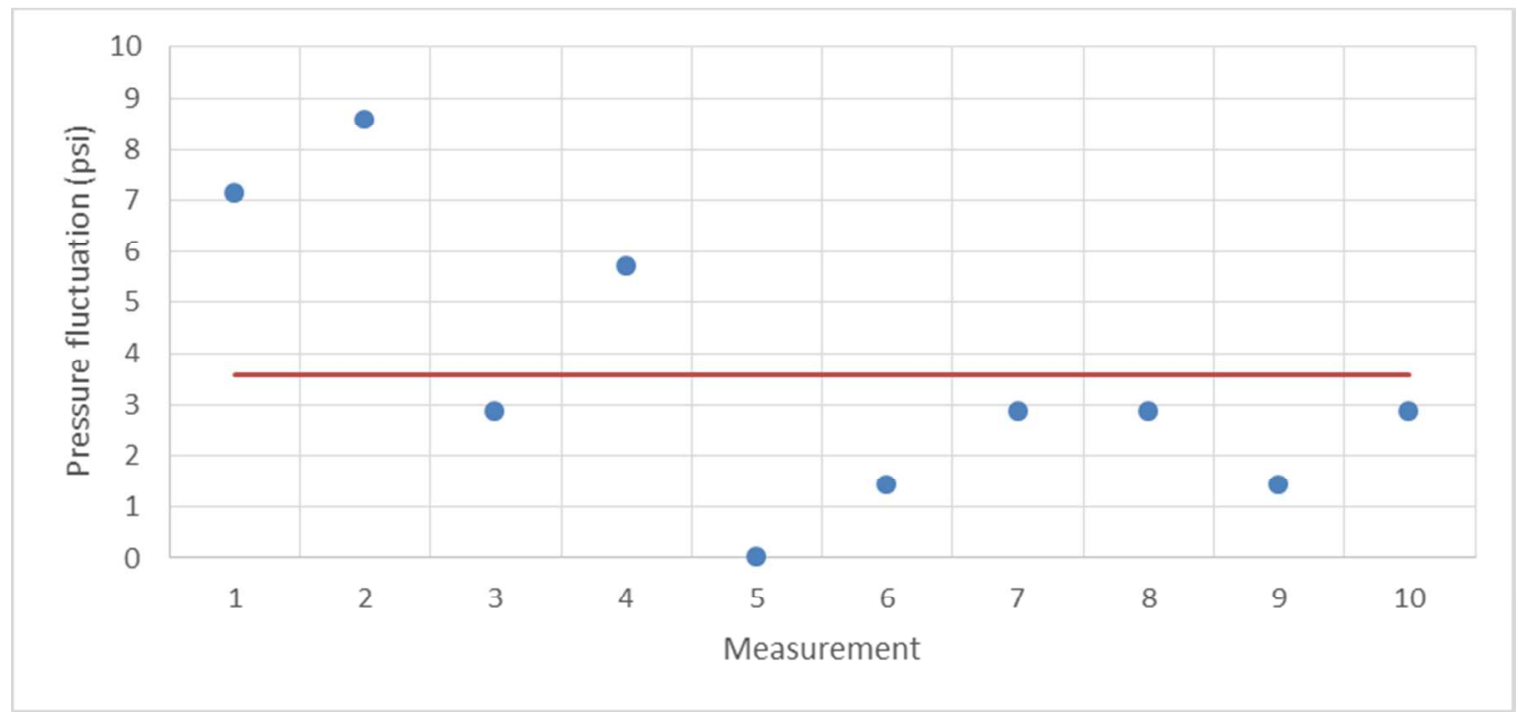

Figure S13. Pressure fluctuation during diversion valve actuation. Red line represents the average value.

\section{Operation of Continous Collection Device}

The Continuous Collection Device (CCD) is composed of two valves (V3, V4) a solvent pump (P4) and the attendant control software. V3 is an 8-port, 2-position valve plumbed with two loops in which reaction product is collected awaiting the result of the sample analysis, V4 is a 3-port, 2-position diversion valve that routes the reaction product to a collection or waste vial. P4 pushes the reaction product from the loop to its destination while simultaneously cleaning the loop. The CCD works in coordination with V2, a 6port, 2-position valve used to take samples of reaction product and the pump, $\mathrm{P} 3$, which 
transfers the sample to analysis. The mechanism of this operation for V2 and V3 is detailed below.
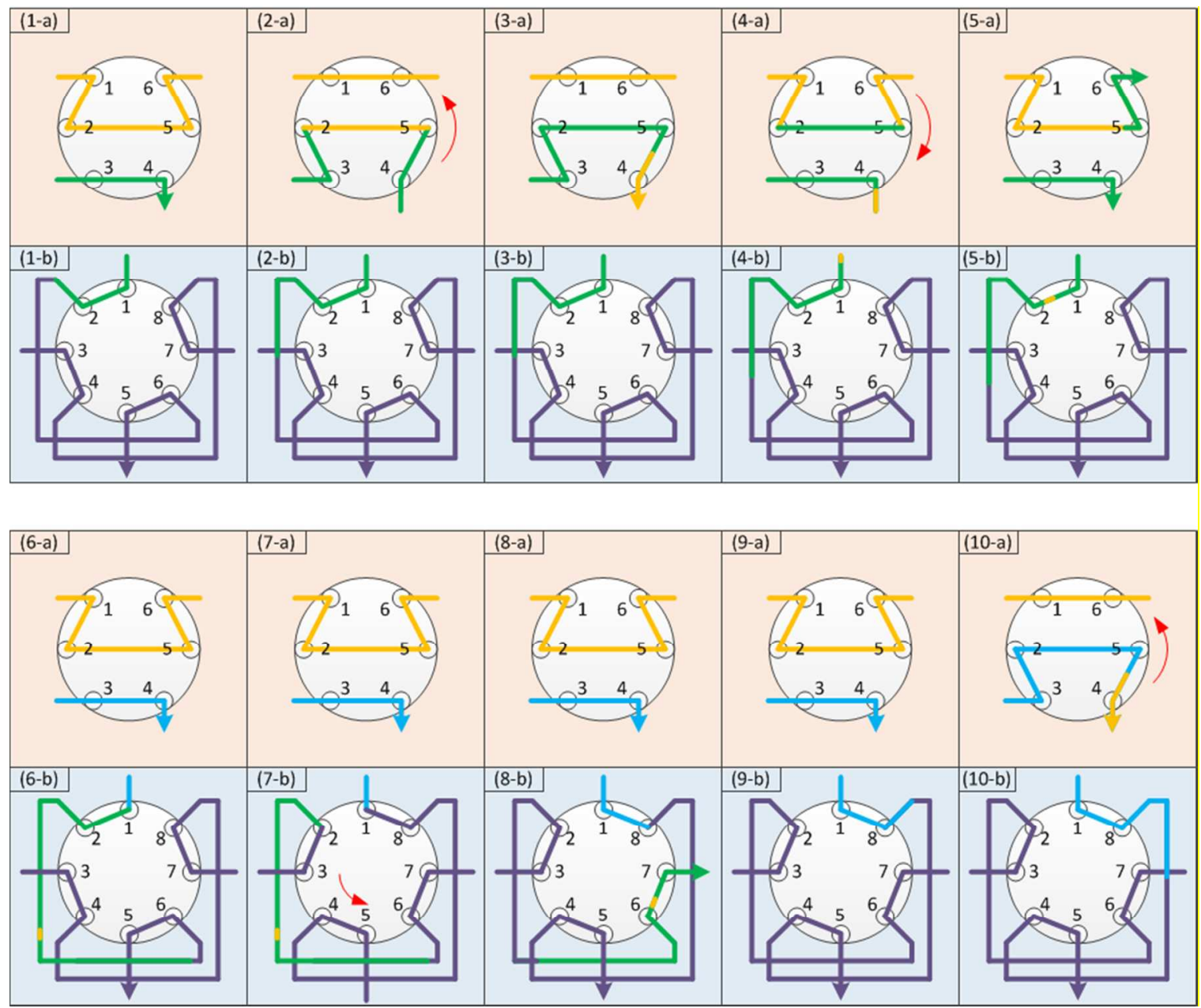

Figure S14. Coordination of sampling valve, V2, and collection valve, V3.

The diversion sequence begins as a metered amount of reaction fluid (green) enters the left loop of the continuous collection valve, V3, (1-b). (2-a) V2, the sampling valve, switches to take a sample as the midpoint of the loop volume to be collected passes-by. (3-a) Process fluid overfills the sampling loop pushing a volume of transfer solvent (yellow) equal to the sample size $(5 \mu \mathrm{L})$ into the process line. (4-a) The sampling valve switches back, and (5-a) the sample plug (i.e., the analyte) is sent for analysis, as (5-b) 
the collection loop continues to fill. (6-b) The collection loop reaches its fill capacity, which is less than the overall loop volume to account for dispersion of the laminar flow, while the analysis of the sample is completed and a decision (i.e., collection or diversion) is made by the software. (7-b) The diversion valve actuates, fluid in the loop is depressurized and removed from the process line and put in communication with the transfer line and pump. (8-b) A second pump (P4) pushing clean transfer solvent flushes the loop material on to its destination, which at the same time, cleans the loop, all connections and the transfer line of detectable product. These integrated functions prevent loop contents from contaminating subsequent isolations. Simultaneously, actuation of $\mathrm{V} 3$ brings the right collection loop into a fluidic communication with the process line and the loop begins to fill with the next volume of material. As the next volume of material (blue) fills the second loop (9-b, 10-b), the midpoint of this volume is sampled (10-a) and the decision-based diversion process is repeated. Repetition of this cycle allows uninterrupted, intelligent collection.

\section{Software and automation}

$\operatorname{MACOS}^{\mathrm{TM}}$ software provides fully automated flow control, coordinating synthetic process functions and analytical results with the Gerstel MPS workstation and Agilent 1200 HPLC.

A custom circuit board sends and receives TTL signals to and from the analytical instruments via a custom cable connected to the serial port on Gerstel liquid handler and the GPIO (general purpose input/output) pins on the circuit board. The liquid handler will not perform an injection until it has received a TTL signal from MACOS $^{\mathrm{TM}}$ software 
indicating that sampling is complete. Correspondingly, $\operatorname{MACOS}^{\mathrm{TM}}$ uses TTL signals from the liquid handler to confirm an analytical action has been completed. If there is no signal error, an analytical result file will be generated. $\operatorname{MACOS}^{\mathrm{TM}}$ searches for the appropriately tagged file in the specified file location for the experimental run. The result will be acquired by $\operatorname{MACOS}^{\mathrm{TM}}$ software upon which it will make the decision to accept, reject or retry the sample.

Software control of $\mathrm{MACOS}^{\mathrm{TM}}$ synthetic hardware devices (pumps, valves, and microwave) is handled by a master/slave relationship. The software monitors device conditions by querying. If a priority condition from the analytical or safety routine has not been met, process actions will be followed according to parameters set by the user prior to initiating the run.

The automation routine can be specifically tailored for a variety of desired process schemes. A sample workflow is illustrated in Figure S15. This figure shows how the software routine integrates $\mathrm{MACOS}^{\mathrm{TM}}$ and analytical hardware to coordinate the synthetic and analytical actions. The work flow on the left is for synthetic unit, and that on the right is for the analytical unit. The two halves communicate through TTL signals. Step1, MACOS ${ }^{\mathrm{TM}}$ takes a sample by switching, waiting, and switching back. The analytical side waits for a TTL signal that denotes the completion of sampling. In step2, $\operatorname{MACOS}^{\mathrm{TM}}$ sends this TTL signal to the liquid handler, which in response moves the injector head to the waste port. Meanwhile, $\operatorname{MACOS}^{\mathrm{TM}}$ activates the solvent pump, flushing product from the sample loop. There are three pump actions, in this case the fluid in front of the analyte sample is sent to waste, pushing the sample plug closer to the injector while minimizing dilution. The liquid handler sends a TTL signal to MACOS ${ }^{\mathrm{TM}}$ 
to acknowledge the completion of this step. At step 3, MACOS ${ }^{\mathrm{TM}}$ sends another TTL signal to the liquid handler to move the injector head to the sample vial. The injector head moves to the vial location descends into the sample vial. The solvent pump is again activated and sample is dispensed into the vial. A TTL signal is sent by the liquid handler, to acknowledge the end of this step. $\operatorname{MACOS}^{\mathrm{TM}}$ software initiates step 4 by sending a TTL signal to move the injector to the waste port again. Here a third volume of solvent is dispensed to clean the line. Step5 is the decision-making step. The analytical unit makes the injection, performs the analysis, and writes a report to the local hard disk. MACOS ${ }^{\mathrm{TM}}$ locates the report by scanning the folder where the report has been deposited. During the search, if the diversion loop is full, the 8-port valve switches to the opposite position to divert the flow into another loop. Once the report is found, software reads it and makes the decision on how to divert the associated loop fluid based on the result. This cycle continues so long as MACOS ${ }^{\mathrm{TM}}$ collects product. Note that the process is fully automated. A pseudo-code of the software is also provided to describe the auto-sample routine. Note that the real numbers involved in the pseudo-code are used in reality and may vary for different setups or experiments. 


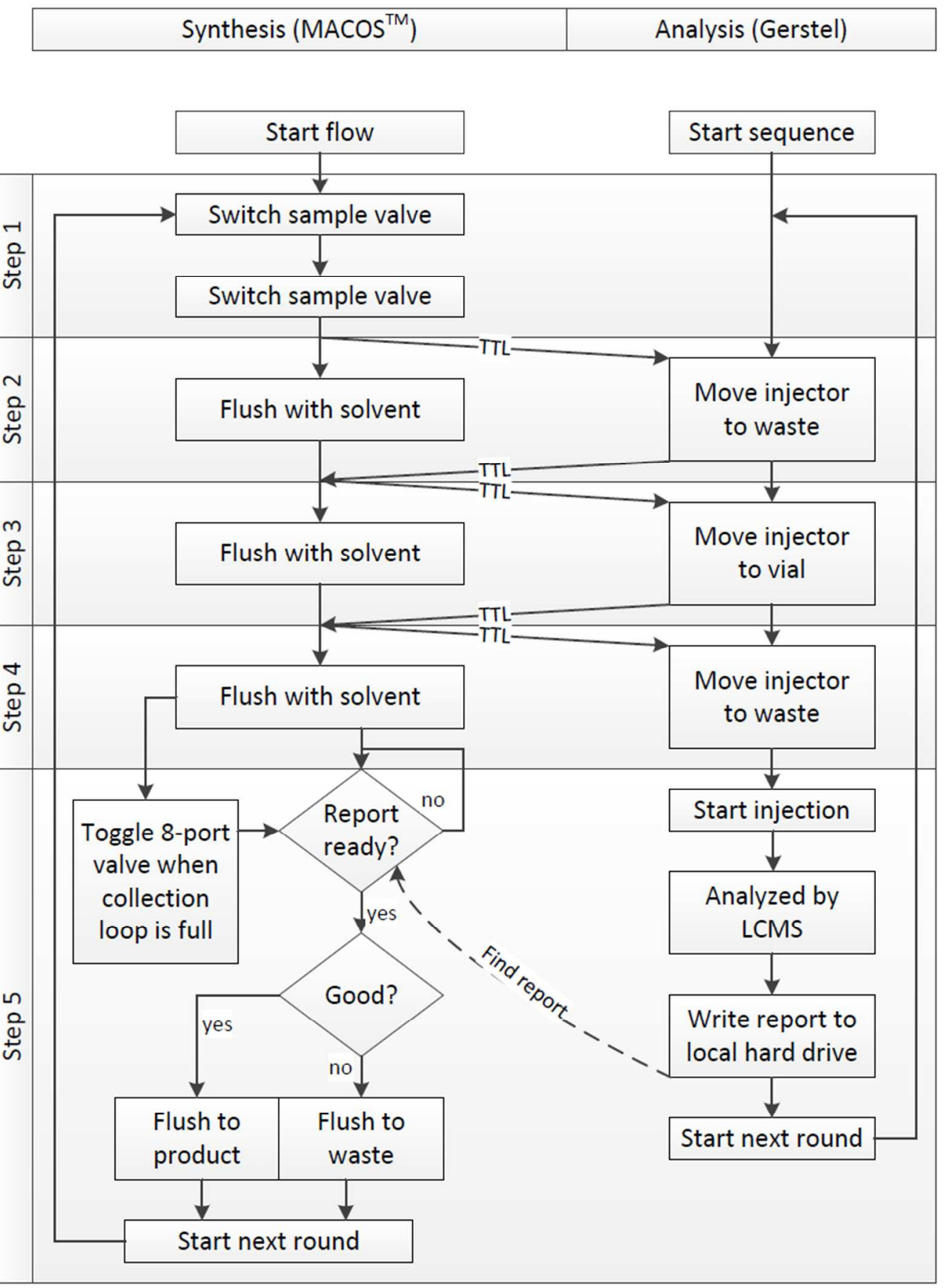

Figure S15. Schematic of $\operatorname{MACOS}^{\mathrm{TM}}$ workflow for smart diversion highlighting communication between synthesis and analysis units. 


\section{Pseudo code}

///// Settings

//// Instruments are defined previously in other sections of the code

//// (e.g. ASpump, ASvalve 8port, ASvalve 4port, TTL in, TTL out)

ASvol = 225;

$/ /$ next sample will be taken at $225 \mu \mathrm{L}$

ASinterval $=500 ; \quad \quad / /$ interval of sampling \& collecting

AScollected $=0 ; \quad$ // cumulatively collected volume

ASstep $=0$;

// step identifier

ASresultThrshd $=1.5 ; \quad$ // criterion of analytical result

///// This function is called repeatedly every second

///// The variable "systemVolume" counts the volume flowed into the system

//////and is updated every second

private void AutoSample(double systemVolume)

\{

if $($ ASstep $==0)$

\{

if ( systemVolume $>=$ ASvol)

\{

sample(25); $\quad$ // sample $25 \mu \mathrm{L}$ (overfilling)

ASstep $=1$;

\}

\}

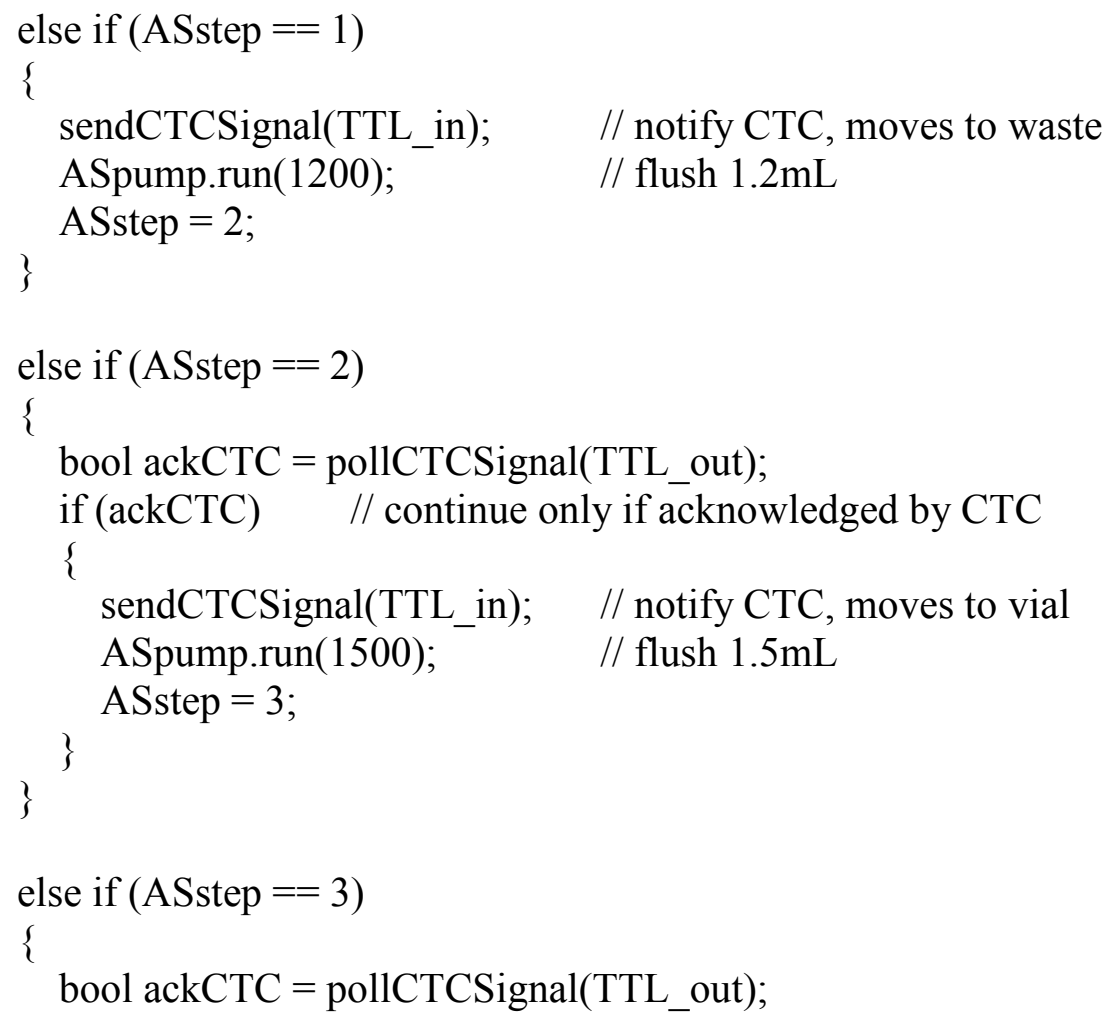




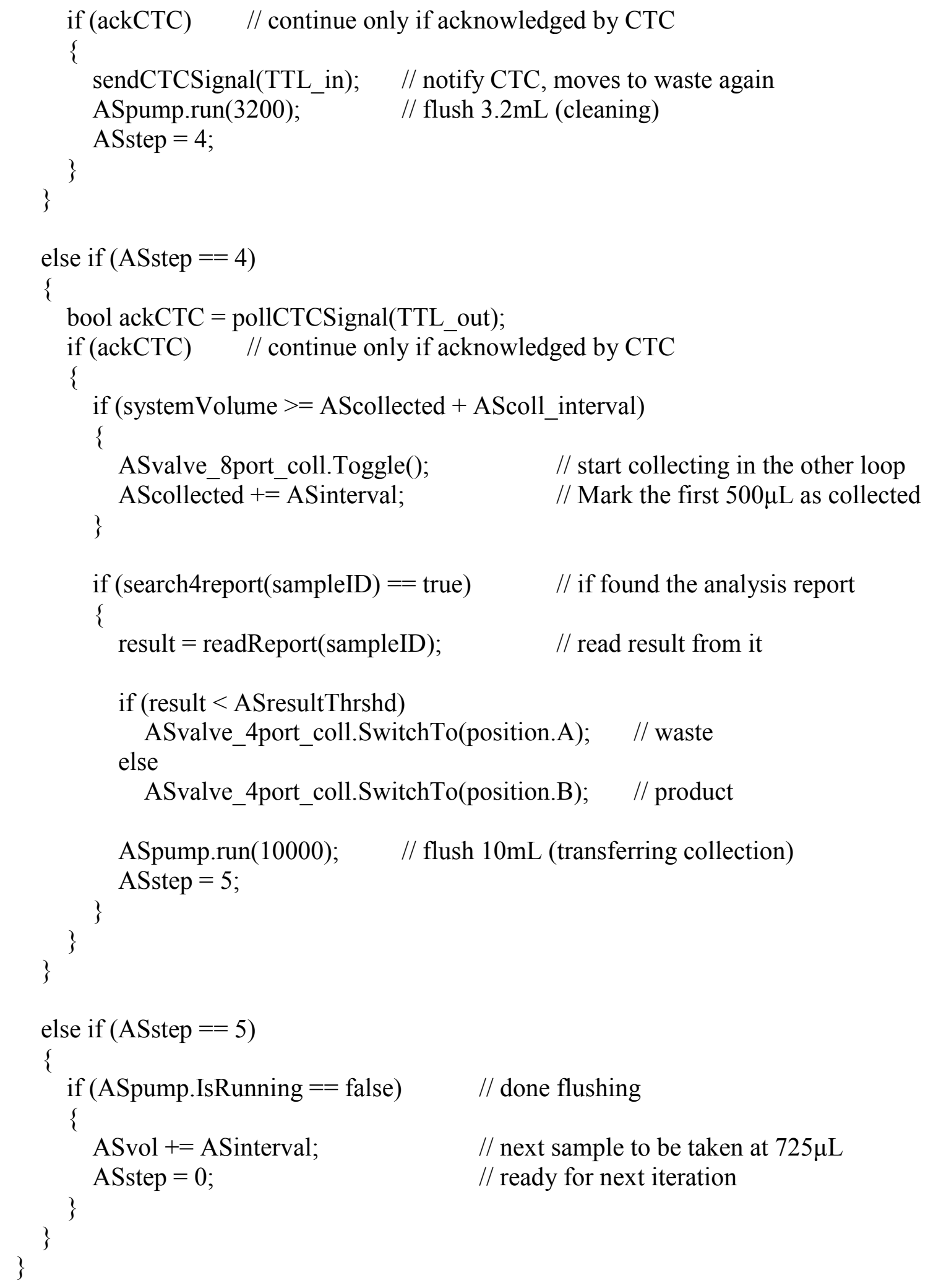




\section{References}

${ }^{1}$ Somerville, K.; Tilley, M.; Li, Guanlong; Mallik, D.; Organ, M. Org. Proc. Res. Dev. $\mathbf{2 0 1 4}, 18,1315-1320$

2 Taylor, G. Proc. R. Soc. London, Ser. A, 1953, 219, 186-203.

${ }^{3}$ Aris, R. Proc. R. Soc. London, Ser. A, 1956, 235, 67-77.

${ }^{4}$ Nagy, K., Shen B., Jamison, T., Jensen, K. Org. Process Res. Dev. 2012, 16, 976-981.

${ }^{5}$ May, S., Johnson, M., Braden, T., Calvin, J., Haeberle, B., Jines, A., Miller, R., Plocharczky, E., Rener, G., Richey, R., Schmid, C., Vaid, R., Yu, H. Org. Process Res. Dev. 2012, 16, 982-1002.

${ }^{6}$ Levenspiel, O. The Chemical Reactor Omnibook; Oregon State University Book Stores, Inc.: Corvallis, OR, 1993. 